OPEN ACCESS

Edited by:

Mourad Ben Said,

University of Manouba, Tunisia

Reviewed by:

Rochelle Haidee Daclan Ybanez, Obihiro University of Agriculture and

Veterinary Medicine, Japan Alexandra Corduneanu,

University of Agricultural Sciences and Veterinary Medicine of Cluj-Napoca, Romania

*Correspondence:

Rosa Estela Quiroz-Castañeda requiroz79@yahoo.com.mx

Specialty section:

This article was submitted to Parasitology,

a section of the journal

Frontiers in Veterinary Science

Received: 16 May 2021

Accepted: 19 July 2021

Published: 17 August 2021

Citation:

Aguilar-Díaz H, Quiroz-Castañeda RE, Cobaxin-Cárdenas M,

Salinas-Estrella E and Amaro-Estrada I

(2021) Advances in the Study of the Tick Cattle Microbiota and the Influence on Vectorial Capacity.

Front. Vet. Sci. 8:710352.

doi: 10.3389/fvets.2021.710352

\section{Advances in the Study of the Tick Cattle Microbiota and the Influence on Vectorial Capacity}

\author{
Hugo Aguilar-Díaz ${ }^{1}$, Rosa Estela Quiroz-Castañeda ${ }^{2 *}$, Mayra Cobaxin-Cárdenas ${ }^{2}$, \\ Elizabeth Salinas-Estrella ${ }^{2}$ and Itzel Amaro-Estrada ${ }^{2}$ \\ ' Unidad de Artropodología del Centro Nacional de Investigación Disciplinaria en Salud Animal e Inocuidad, Instituto Nacional \\ de Investigaciones Forestales, Agrícolas y Pecuarias, Morelos, Mexico, ${ }^{2}$ Unidad de Anaplasmosis del Centro Nacional de \\ Investigación Disciplinaria en Salud Animal e Inocuidad, Instituto Nacional de Investigaciones Forestales, Agrícolas y \\ Pecuarias, Morelos, Mexico
}

The information from the tick cattle microbiota suggests that the microbial populations may modulate a successful infection process of the tick-borne pathogens. Therefore, there is a need to know the microbial population and their interactions. In this mini-review, we present several examples of how microbiota regulates the survival of pathogens inside the tick and contributes to fitness, adaptation, and tick immunity, among others. The communication between the tick microbiota and the host microbiota is vital to understanding the pathogen transmission process. As part of the tick microbiota, the pathogen interacts with different microbial populations, including the microorganisms of the host microbiota. These interactions comprise a microsystem that regulates the vectorial capacity involved in tick-borne diseases. The knowledge we have about the vectorial capacity contributes to a better understanding of tick-borne pathogens. Additionally, using approaches based on multi-omics strategies applied to studying the microbiota and its microbiome allows the development of strategies to control ticks. The results derived from those studies reveal the dynamics of the microbiota and potential targets for anti-tick vaccine development. In this context, the anti-microbiota vaccines have emerged as an alternative with a good prognosis. Some strategies developed to control other arthropods vectors, such as paratransgenesis, could control ticks and tick-borne diseases.

Keywords: bovine host, vector of transmission, metagenomics, microbiota, microbiome, vectorial capacity, ticks

\section{INTRODUCTION}

Infectious diseases have been one of the main restrictions worldwide for animal production improvement, with significant economic losses. The significant risk that endangers animal health is the arthropod vector and the pathogens they transmit and those that have been controlled or eradicated, and after a while, they reemerge. Ticks are hematophagous ectoparasites, the main biological vectors of numerous infectious diseases (tick-borne diseases) (1-3) (Figure 1).

Although the study of the tick microbiota and the biological processes in which it participates are still in progress to date, there is not enough information about its role in the vectorial capacity, infection, and pathogen transmission. This study opens up a research field to study and elucidate new targets for developing drugs/vaccines to prevent diseases that affect animals, including humans. The development of anti-tick vaccines based on the microbiota represents a 
promising approach to control tick infestations. In this regard, the study of the tick microbiota contributes to elucidate the interactions that may be influencing vital processes and then avoiding the transmission of pathogens $(4,5)$. However, there is much work to obtain an effective drug or vaccine to control tick-borne diseases and the pathogens that cause them.

\section{A BRIEF OVERVIEW OF TICK-BORNE DISEASES IN CATTLE}

During the last years, the number of tick-borne diseases caused by bacteria Anaplasma, Ehrlichia, and Coxiella has increased, affecting livestock productivity (6). Anaplasma marginale causes bovine anaplasmosis resulting in economic losses to the cattle industry due to a significant reduction of beef and dairy production (7). More recently, some coinfections with piroplasmas and other Anaplasmataceae reported in cattle and buffaloes show the circulation of diverse genotypes of $A$. marginale worldwide (8).

So far, there exist more than 20 genomes and draft genomes of A. marginale, whose content could contribute to the identification and participation of the genes involved in the microbiota-vector-host interaction (9-11).

Ehrlichia ruminantium is transmitted by Amblyomma and causes Heartwater, the most important livestock disease in Africa and the Caribbean, while the emerging Ehrlichia minasensis also infects cattle (12-14). As an example of the intrinsic interaction between pathogen and the host cell, molecular studies reveal that the genome of Ehrlichia sp. has lost genes associated with metabolism whose activities are covered by the host cell (15). In the genomic context, only a few genomes are available for Ehrlichia species $(16,17)$.

In European livestock, coxiellosis, caused by Coxiella burnetii and transmitted by Hyalomma spp. and Rhipicephalus spp. has a significant prevalence in the Mediterranean countries (18).

At the moment, there exist more than 75 Coxiella genomes reported that contain a large number of genes participating in adhesion, invasion, intracellular trafficking, host-cell modulation, and detoxification (19).

In Africa, Ben Said et al. (20) interestingly identified the spirochaetes, Borrelia burgdorferi, in goats, sheep, camels, and cattle, transmitted by Rhipicephalus and Hyalomma. The recent molecular detection of Rickettsia spp. and C. burnetii in cattle and water buffalo in Luzon Island of the Philippines reveals the potential zoonotic transmission mediated by Rhipicephalus microplus (21).

Hemoplasmosis is not strictly considered a tick-borne disease; however, Mycoplasma wenyonii and Candidatus Mycoplasma haemobos reported in cattle are probably transmitted by ticks (22-25).

\section{CATTLE TICK MICROBIOTA INTERACTIONS AND VECTORIAL CAPACITY}

The microbial communities that comprise the tick microbiota include pathogens, symbionts, and commensals acting as a dynamic and integrative microecosystem, changing in time and scale, that interact into a macrosystem that includes the host $(26,27)$. Additionally, external factors modulate the diversity of the microbiota: temperature, humidity, geographic location, sex and species, blood intake from the vertebrate host, or even the physical location inside the organs of the tick (gut, ovaries, salivary glands) $(28,29)$.

Interestingly, this microbiota also contributes to fitness, nutritional adaptation, development, and reproduction and is also involved in establishing pathogens inside the tick $(30,31)$.

Currently, there exists a close relationship between pathogens and the tick microbiota, which affects their vectorial capacity. Also, colonization, replication, or maturation of an infective form of a pathogen depends mainly on the microbiota composition.

During the blood intake, the hematophagous vector can acquire pathogens from an infected host that transmit to a new host. This is known as vector competence, the ability of a vector to maintain the pathogen development until its transmission to a new host. Vector competence is a component of vectorial capacity that comprises the interactions between vector-pathogen and vector-host, influenced by behavioral and environmental factors such as vector density, longevity, host preference, and feeding habits (32).

In tick cattle, the study of interaction microbiota-vector is still scarce. However, the results reported in other species could be applied as alternatives to identify new targets to control ticks. In addition, after blood intake, some complex interactions are carried out by the triad microbiota-vector-host involved in the pathogen transmission from one host to another (Figure 2). In this regard, the first interaction occurs between the tick and the microbiota of the bovine skin, in which several families of bacteria are present, including Corynebacteriaceae and Staphylococcaceae (teat skin), and Firmicutes, Spirochaetae, Bacteroidetes, and Actinobacteria (interdigital skin) $(33,34)$ (Figure 2). The microorganisms penetrate from the surface of the skin host deeper into the dermis and might be inducing local immunomodulation (35). For instance, the bacteria Prevotella sp. and Neisseria sp., usually found in the skin and mucosal surface of the host, respectively, have been identified in the midgut of I. ricinus blood-fed females, regardless of the time point of the blood feeding course $(36,37)$. In contrast, Miranda-Miranda et al. $(38,39)$ found that bacteria Staphylococcus saprophyticus and Staphylococcus xylosus, located in the skin of bovine, can produce a lethal infection in fully engorged female ticks that lose the ability to oviposit and lead them eventually to death. These findings show that the composition of the microbiota does not always favor tick infestations and pathogen transmission.

A second interaction occurs when the microbiota of the salivary glands is in contact with the pathogen that enters the tick. Here, the microbiota has an essential interaction with the pathogens, as in Amblyomma americanum, where the Coxiellarelated symbionts in the salivary glands impair the transmission of Ehrlichia chaffeensis (40). These studies illustrate the pathogen interactions occurring in the salivary glands and highlight the role of the tick microbiota that regulates pathogen growth. Alternatively, during the pathogen inoculation, the microbiota of the saliva interacts with the host's components regulating the pathogen passage from the salivary glands to a new bite site. 


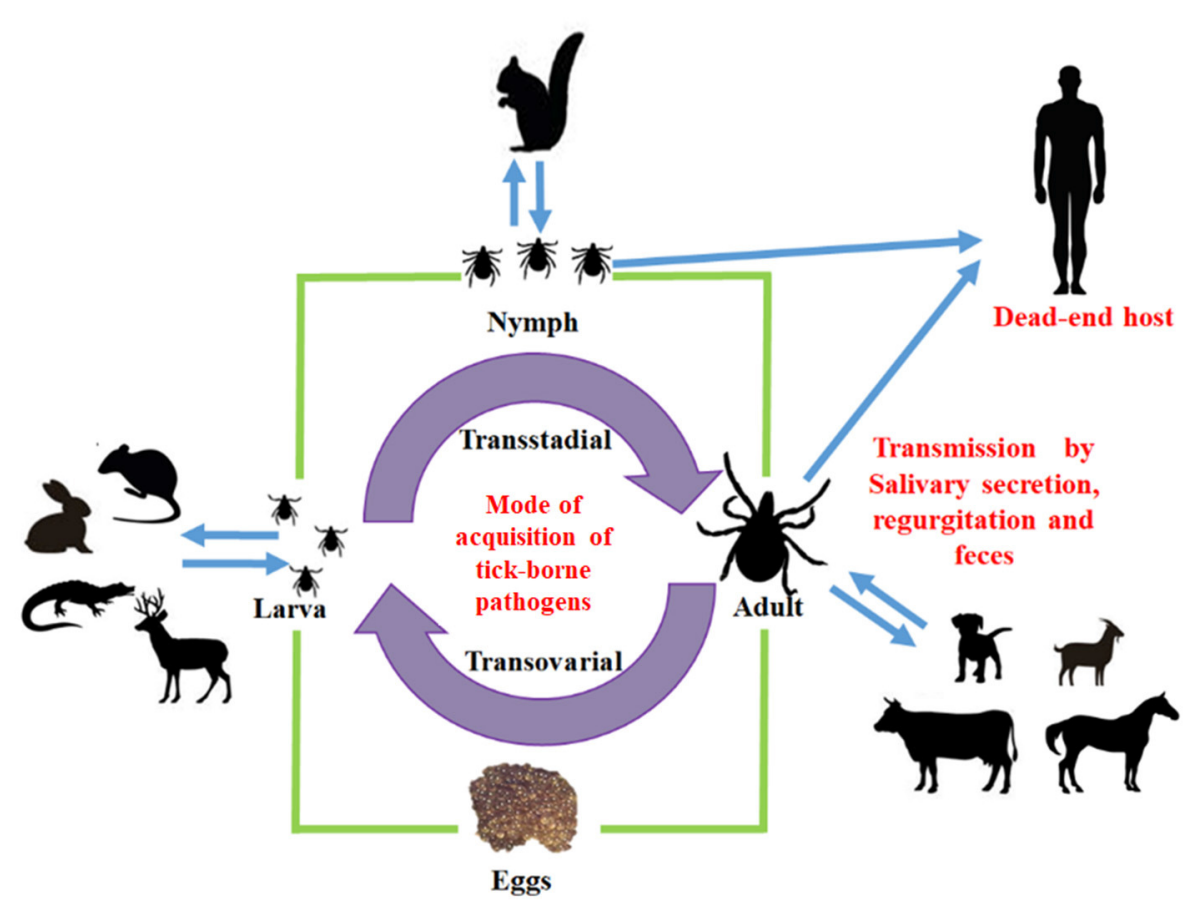

FIGURE 1 | Mode of acquisition of tick-borne pathogens. The different stages of tick (larva, nymph, and adult) may transmit bacteria to intermediate or final host.

The tick gut microbiota possesses different microorganisms that participate in metabolic and digestive processes. In this tissue, the blood and the pathogens ingested interact and alter the microbiota, and a third interaction occurs. In such a way, the altered microbiota, which usually provides cofactors, vitamins $B$, and folate (e.g., Coxiella, Francisella, Rickettsia, respectively), may affect the tick's development, as occurs in Ornithodoros moubata, where antibiotic-based elimination of Francisella endosymbionts compromise the nutritional status of vitamin $\mathrm{B}$, which results in some anomalies in tick development and hampers nymph growth and molting to adults (41). Another role of the microbiota is to regulate the pathogen colonization of the tick gut. For example, in Ixodes scapularis a gut microbiota composed of a high abundance of Rickettsia, Thioclava, and Delftia, and a low abundance of Aquabacterium, Brevibacterium, and Novosphingobium may influence B. burgdorferi colonization negatively (42).

Once established, the pathogens cross the peritrophic membrane and the gut barrier to disseminating to other tissues through the hemolymph. Here, the presence of hemocytes and effector molecules of the tick immune response represents a hostile environment for the pathogen; however, the information about the role of the microbiota of the hemolymph and its interaction is scarce except for the finding of Staphylococcus aureus in Rhipicephalus decoloratus and Rhipicephalus geigy, which were isolated from the adult females hemolymph (43).

Finally, the pathogen could likely take two routes. The first, pathogens redirect to the salivary glands and transmit to a new host, and the second, pathogens transmit by transovarial transmission. In both cases, the pathogen would be interacting with the microbiota of the tissue.

Although the information about the effect of tick-borne pathogens and microbiota is scarce, the report of co-occurences in epidemiological studies has suggested the impact of pathogens on the tick and vice versa (27). For example, the microbial community in the tick gut is related to the virulence and proliferation of Anaplasma sp. and Babesia sp. (27). Adegoke et al. (44) reported that microbial diversity and composition of $R$. microplus decreases when infected with Theileria sp.; this phenomenon is called pathogen-induced dysbiosis. A similar effect occurs in D. andersoni where endosymbiont Francisella sp. increases the successful pathogenic colonization of Francisella novicida and $A$. marginale $(45,46)$.

To address the potential role of the I. scapularis gut bacteria in modulating B. burgdorferi, (42) compared dysbiosed larvae and larvae fed on gentamicin-treated mice. They found that both types of larvae significantly increased engorgement weights and decreased B. burgdorferi colonization, suggesting that tick-altered gut microbiota impairs pathogen colonization.

On the other hand, the induction of $I$. scapularis antifreeze glycoprotein (IAFGP) occurs in the presence of A. phagocytophilum, which sequesters IAFGP to alter its accumulation in the tick midgut to inhibit the development of biofilms, thus allowing the colonization and establishment of the vector $(47,48)$.

Understanding how microbial diversity of ticks changes in the presence of pathogens could help propose better strategies 


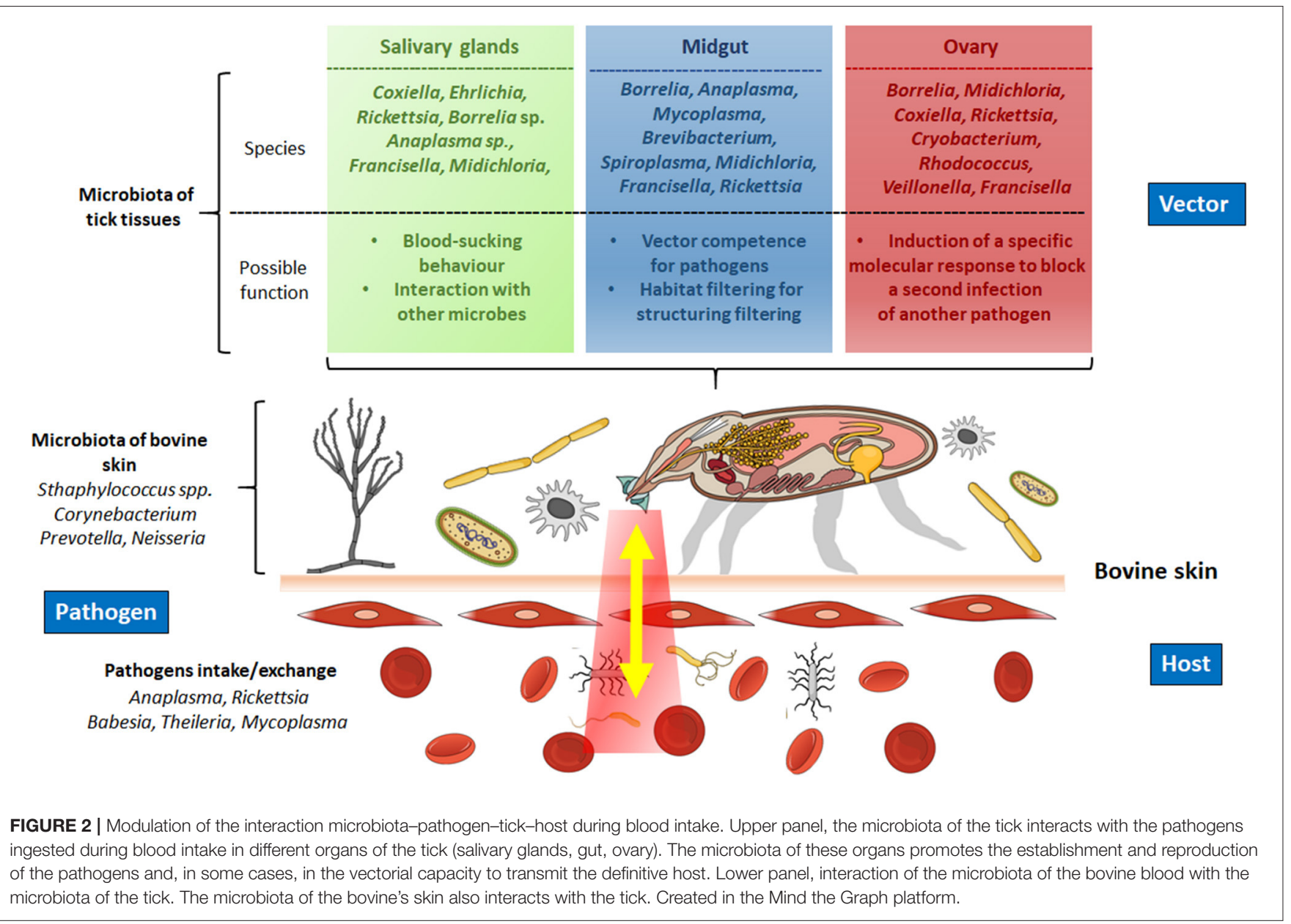

to prevent colonization and establishment in the vector (49). Also, the interaction of the tick microbiota with the host microbiota affects vector competence; therefore, the elucidation of the mechanisms involved in these interactions allows the identification of molecular drivers for tick-borne disease (50).

\section{METAGENOMIC APPROACHES TO STUDY PATHOGENS TRANSMITTED BY TICKS}

So far, we have known that ticks have a great capacity to transmit various pathogens that cause different diseases in cattle. However, the function of the microbiota within the tick life cycle is still unclear due to the significant limitation of their isolation, cultivation, and propagation in culture media and the difficulty of infesting animals with ticks under controlled experimental conditions $(30,51)$. Because of these drawbacks, the use of next-generation sequencing, molecular techniques, and or multiomics technologies represents valuable tools in the study of the tick microbiota and its microbiome.

In this regard, the use of $16 \mathrm{~S}$ rRNA gene-targeted metagenomics provides new insights into tick-borne pathogens (52). The use of this technique has allowed the identification of bacteria in ticks such as $R$. microplus, Hyalomma anatolicum, and Haemaphysalis montgomery from cows, where Ralstonia, Staphylococcus, and Francisella were some genera detected (53). Metagenomics studies show that the microbiota of $R$. microplus captured in a rural area in Peru has 147 bacterial genera (54). In contrast, the sequencing of internal tissue and salivary glands from unfed larvae and female ticks of I. ricinus revealed commensal bacteria, endosymbionts, and several pathogenic microorganisms $(54,55)$. Also, metagenomic analysis of Ixodes persulcatus and Dermacentor nuttalli revealed 10 predominant genera of cattle pathogens and also coinfections (56).

A deeper metagenomic study elucidated the taxonomic and functional profiles of the microbiome of female and male ticks, Ixodes ovatus, I. persulcatus, and Amblyomma variegatum (57). The results of this study showed significant differences in the abundance of genes involved in metabolic pathways between female and male ticks of the same species, suggesting their different lifestyles exert and sex-specific evolutionary pressure independently of the phenomes and microbiomes of the tick gut microbiota.

Complementary information to metagenomics studies in microbiota derives from other multi-omics strategies such as metabolomics, transcriptomics, and proteomics that contribute 
to advancing knowledge of pathogens and their interactions with the host and vector (58), as in the case of the studies that show diverse components of tick saliva are capable of modulating host immune response through the binding to cell receptors and regulating the secretion of cytokines, chemokines, and interleukins (35). However, we propose that tick microbiota requires an interdisciplinary approach, where the metagenomics study combined with other multi-omics tools complements the results to give a complete vision of the microbiota-vectorhost interactions.

\section{PARATRANSGENESIS AS A STRATEGY TO CONTROL TICKS}

Based on the progress of metagenomics study applied to identify microbiota in other arthropods vectors, such as Aedes, Culex, and Anopheles, strategies such as paratransgenesis could control tick infestations. This strategy is an innovative method with a good prognosis in the vectors control. This strategy requires knowledge of the tick microbiota. After the symbiont microorganisms' identification, they are isolated and genetically transformed to generate bacteria capable of expressing the specific inhibitory molecules and then reintroduced to obstruct vital biological processes of the vector. In this regard, some reports suggest that the interference of crucial processes such as ovogenesis and vitellogenesis decreases the fecundity, development, and hatching rates, which results in the inhibition of the vector population growth (59). Similarly, another target is the transformation of bacteria that interfere with the digestive capacity, leading to reduced vector reproduction and preventing pathogen dissemination (59). Adopting strategies based on metagenomic studies allows identifying symbiont bacteria in the tick microbiota that can be genetically modified and cultivated with higher efficiency, which overcomes the need to produce genetically modified vectors $(60,61)$.

\section{MICROBIAL TARGETS: ANTI-TICK VACCINES}

In response to the presence of acaricide-resistant populations, several vaccine proposals were developed in the last years, including the use of antigens such as Gavac, a vaccine based on protein Bm86 (62). The formulation of cocktail anti-tick vaccines has been reported using combinations of antigens, like Bm91 (R. microplus); subolesin 4E6 (I. scapularis); serpins rRAS2 and rRAS-4 (R. appendiculatus); and glutathione S-transferase rGST-Av (A. variegatum), among others (63).

Currently, the use of experimental anti-tick vaccines targeting the microbiota reveals that modifications in the microbial populations of the gut could alter essential processes in a tick. For instance, the use of this experimental anti-tick vaccine developed after a functional metagenomic analysis shows that immunization of $\alpha$-1,3-galactosyltransferase-deficient mice with Escherichia coli BL21 induces the production of anti-E. coli and anti- $\alpha-$ Gal IgM and IgG associated with mortality of I. ricinus nymphs during feeding, which concurred with the abundance of $\alpha$-1,3-galactosyltransferase genes and possibly $\alpha$-Gal identified by tick microbiome metagenomics analysis (5).

Finally, the search for new vaccine targets should encompass those proteins of the tick immune response involved in the tolerance of microbial populations (tick microbiota), like different proteins identified by immunoinformatic analysis (64).

\section{CONCLUSIONS}

Here, we show a general view of different approaches for studying the tick microbiota and other arthropod vectors, intending to integrate the current knowledge and present new alternatives for tick control. From our perspective, in this mini-review, we include recent results that show that the more knowledge and manipulation we have of the microbiota, we get closer to new vaccine development.

The study of the tick microbiota based on metagenomics approaches allows identifying microorganisms and can elucidate the genes that shape the microbiome.

Although the microbiota participates in biological processes like adaptation, development, reproduction, defense against environmental stress, and immunity, information about the interactions and mechanisms involved in vectorial capacity is still scarce and, at the same time, is a field with potential for the identification of vaccine targets.

Considering that many biological interactions function as holobionts (host organism and its associated microbial community) and that, in turn, holobionts make up a more extensive consortium, it is impossible to think that the tick microbiota and tick-borne pathogens are isolated processes.

Undoubtedly, deciphering the interaction of the tick and host-microbiota and how they communicate will provide invaluable information to develop novel strategies for controlling ticks and vector-borne pathogens, like those anti-microbiota and anti-tick vaccine candidates that could benefit animal health and provide acaricide-free environments.

\section{AUTHOR CONTRIBUTIONS}

REQ-C and HA-D: conceptualization. REQ-C, HA-D, ES-E, $\mathrm{MC}-\mathrm{C}$, and IA-E: investigation and original draft preparation and writing and draft preparation. REQ-C and HA-D: review and editing. All the authors have read and agreed to the published version of the manuscript.

\section{ACKNOWLEDGMENTS}

IAE acknowledge to Project CONACyT CB 252577. 


\section{REFERENCES}

1. Estrada-Peña A, Salman M. Current limitations in the control and spread of ticks that affect livestock: a review. Agric. (2013) 3:22135. doi: 10.3390/agriculture3020221

2. Cabezas-Cruz A, Espinosa PJ, Obregón DA, Alberdi P, de la Fuente J. Ixodes scapularis tick cells control anaplasma phagocytophilum infection by increasing the synthesis of phosphoenolpyruvate from tyrosine. Front Cell Infect Microbiol. (2017) 7:375. doi: 10.3389/fcimb.2017.00375

3. Saracho-Bottero MN, Tarragona EL, Sebastian PS, Venzal JM, Mangold AJ, Guglielmone AA, et al. Ticks infesting cattle and humans in the Yungas Biogeographic Province of Argentina, with notes on the presence of tick-borne bacteria. Exp Appl Acarol. (2018) 74:107-16. doi: 10.1007/s10493-018-0208-4

4. Bhowmick B, Han Q. Understanding tick biology and its implications in antitick and transmission blocking vaccines against tick-borne pathogens. Front Vet Sci. (2020) 7:319. doi: 10.3389/fvets.2020.00319

5. Mateos-Hernández L, Obregón D, Maye J, Borneres J, Versille N, de la Fuente $\mathrm{J}$, et al. Anti-tick microbiota vaccine impacts ixodes ricinus performance during feeding. Vaccines. (2020) 8:702. doi: 10.3390/vaccines 8040702

6. Parola P, Paddock CD, Raoult D. Tick-borne rickettsioses around the world: emerging diseases challenging old concepts. Clin Microbiol Rev. (2005) 18:719-56. doi: 10.1128/CMR.18.4.719-756.2005

7. Kocan KM, de la Fuente J, Blouin EF, Coetzee JF, Ewing SA. The natural history of Anaplasma marginale. Vet Parasitol. (2010) 167:95107. doi: 10.1016/j.vetpar.2009.09.012

8. Al-Hosary A, Răileanu C, Tauchmann O, Fischer S, Nijhof AM, Silaghi C. Epidemiology and genotyping of Anaplasma marginale and co-infection with piroplasms and other Anaplasmataceae in cattle and buffaloes from Egypt. Parasit Vectors. (2020) 13:495. doi: 10.1186/s13071-020-04372-Z

9. Brayton KA, Kappmeyer LS, Herndon DR, Dark MJ, Tibbals DL, Palmer GH, et al. Complete genome sequencing of Anaplasma marginale reveals that the surface is skewed to two superfamilies of outer membrane proteins. Proc Natl Acad Sci USA. (2005) 102:844-9. doi: 10.1073/pnas.0406656102

10. Quiroz Castañeda RE, Amaro-Estrada I, Martínez-Ocampo F, RodríguezCamarillo S, Dantán González E, Cobaxin-Cárdenas M, et al. Draft genome sequence of anaplasma marginale strain Mex-01-001-01, a Mexican strain that causes bovine anaplasmosis. Microbiol Resour Announc. (2018) 7:e0110118. doi: 10.1128/MRA.01101-18

11. Martínez-Ocampo F, Quiroz-Castañeda RE, Amaro-Estrada I, Cobaxin Cárdenas M, Dantán-González E, Rodríguez-Camarillo S. Draft genome sequences of Anaplasma marginale Strains MEX-15-099-01 and MEX-31-09601, two Mexican isolates with different degrees of virulence. Microbiol Resour Announc. (2019) 8:e01184. doi: 10.1128/MRA.01184-19

12. Allsopp BA. Heartwater - Ehrlichia ruminantium infection Aetiology Antemortem diagnosis pathology and post-mortem diagnosis. Rev Sci Tech. (2015) 34:557-68. doi: 10.20506/rst.34.2.2379

13. Cabezas-Cruz A, Zweygarth E, Aguiar DM. Ehrlichia minasensis, an old demon with a new name. Ticks Tick Borne Dis. (2019) 10:8289. doi: 10.1016/j.ttbdis.2019.03.018

14. Peter SG, Aboge GO, Kariuki HW, Kanduma EG, Gakuya DW, Maingi N, et al. Molecular prevalence of emerging Anaplasma and Ehrlichia pathogens in apparently healthy dairy cattle in peri-urban Nairobi, Kenya. BMC Vet Res. (2020) 16:364. doi: 10.1186/s12917-020-02584-0

15. McBride JW, Walker DH. Molecular and cellular pathobiology of Ehrlichia infection: targets for new therapeutics and immunomodulation strategies. Expert Rev Mol Med. (2011) 13:e3. doi: 10.1017/S1462399410001730

16. Collins NE, Liebenberg J, de Villiers EP, Brayton KA, Louw E, Pretorius A, et al. The genome of the heartwater agent Ehrlichia ruminantium contains multiple tandem repeats of actively variable copy number. Proc Natl Acad Sci USA. (2005) 102:838-43. doi: 10.1073/pnas.0406633102

17. Aguiar D, João A, Luciano N, Emilie B, Alejandro C-C. Complete genome sequence of an ehrlichia minasensis strain isolated from cattle. Microbiol Resour Announc. (2021) 8:e00161-e00119. doi: 10.1128/MRA.00161-19

18. Körner S, Makert GR, Ulbert S, Pfeffer M, Mertens-Scholz K. The prevalence of coxiella burnetii in hard ticks in europe and their role in q fever transmission revisited-a systematic review. Front Vet Sci. (2021) 8:233. doi: $10.3389 /$ fvets. 2021.655715
19. Hemsley CM, O’Neill PA, Essex-Lopresti A, Norville IH, Atkins TP, Titball RW. Extensive genome analysis of Coxiella burnetii reveals limited evolution within genomic groups. BMC Genomics. (2019) 20:441. doi: 10.1186/s12864-019-5833-8

20. Ben Said M, Belkahia H, Alberti A, Abdi K, Zhioua M, Daaloul-Jedidi M, et al. First molecular evidence of Borrelia burgdorferi sensu lato in goats, sheep, cattle and camels in Tunisia. Ann Agric Environ Med. (2016) 23:442-7. doi: 10.5604/12321966.1219184

21. Galay RL, Talactac MR, Ambita-Salem BV, Chu DMM, Dela Costa LMO, Salangsang CMA, et al. Molecular detection of Rickettsia Spp. and coxiella burnetii in cattle, water buffalo, and rhipicephalus (Boophilus) microplus ticks in luzon island of the philippines. Trop. Med. Infect. Dis. (2020) 5:54. doi: 10.3390/tropicalmed5020054

22. Meli ML, Willi B, Dreher UM, Cattori V, Knubben-Schweizer G, Nuss K, et al. Identification, molecular characterization, and occurrence of two bovine hemoplasma species in Swiss cattle and development of real-time TaqMan quantitative PCR assays for diagnosis of bovine hemoplasma infections. J Clin Microbiol. (2010) 48:3563-8. doi: 10.1128/JCM.02224-09

23. Martínez-Ocampo F, Rodríguez-Camarillo SD, Amaro-Estrada I, QuirozCastañeda RE. Draft genome sequence of "Candidatus Mycoplasma haemobos," a hemotropic mycoplasma identified in cattle in Mexico. Genome Announc. (2016) 4:1-2. doi: 10.1128/genomeA.00656-16

24. Souza UA, Oberrather K, Fagundes-Moreira R, Almeida BA, de Valle S, de F, et al. First molecular detection of Mycoplasma ovis (Hemotropic mycoplasmas) from Sheep in Brazil. Rev Bras Parasitol Veterinária. (2019) 28:360-6. doi: 10.1590/s1984-29612019022

25. Quiroz Castañeda RE, Aragón KM, Diaz HA, Preciado de la Torre JF. Molecular detection of bovine hemotrophic mycoplasmas in Mexico. Rev del Cent Investig la Univ la Salle. (2020) 13:67-82. doi: 10.26457/recein.v13i52.2183

26. Berg G, Rybakova D, Fischer D, Cernava T, Vergès M-CC, Charles T, et al. Microbiome definition re-visited: old concepts and new challenges. Microbiome. (2020) 8:103. doi: 10.1186/s40168-020-00875-0

27. Bonnet SI, Pollet T. Update on the intricate tango between tick microbiomes and tick-borne pathogens. Parasite Immunol. (2021) 43:e12813. doi: 10.1111/pim.12813

28. Gurfield N, Grewal S, Cua LS, Torres PJ, Kelley ST. Endosymbiont interference and microbial diversity of the Pacific coast tick, Dermacentor occidentalis, in San Diego County, California. PeerJ. (2017) 5:e3202. doi: $10.7717 /$ peerj.3202

29. Brinkerhoff RJ, Clark C, Ocasio K, Gauthier DT, Hynes WL. Factors affecting the microbiome of Ixodes scapularis and Amblyomma americanum. PLoS ONE. (2020) 15:e232398. doi: 10.1371/journal.pone.0232398

30. Bonnet SI, Binetruy F, Hernández-Jarguín AM, Duron O. The tick microbiome: why non-pathogenic microorganisms matter in tick biology and pathogen transmission. Front Cell Infect Microbiol. (2017) 7:236. doi: $10.3389 /$ fcimb. 2017.00236

31. Pollet T, Sprong H, Lejal E, Krawczyk AI, Moutailler S, Cosson J-F, et al. The scale affects our view on the identification and distribution of microbial communities in ticks. Parasit Vectors. (2020) 13:36. doi: 10.1186/s13071-020-3908-7

32. Beerntsen BT, James AA, Christensen BM. Genetics of mosquito vector competence. Microbiol Mol Biol Rev. (2000) 64:11537. doi: 10.1128/MMBR.64.1.115-137.2000

33. Rodrigues Hoffmann A. The cutaneous ecosystem: the roles of the skin microbiome in health and its association with inflammatory skin conditions in humans and animals. Vet Dermatol. (2017) 28:60-e15. doi: 10.1111/vde. 12408

34. Porcellato D, Meisal R, Bombelli A, Narvhus JA. A core microbiota dominates a rich microbial diversity in the bovine udder and may indicate presence of dysbiosis. Sci Rep. (2020) 10:21608. doi: 10.1038/s41598-020-77054-6

35. Boulanger $\mathrm{N}$, Wikel $\mathrm{S}$. Induced transient immune tolerance in ticks and vertebrate host: a keystone of tick-borne diseases?. Front Immunol. (2021) 12:284. doi: 10.3389/fimmu.2021.625993

36. Elliott D, Michael W, Buckley CMF, Spratt DA. Cultivable oral microbiota of domestic dogs. J Clin Microbiol. (2005) 43:5470-6. doi: 10.1128/JCM.43.11.5470-5476.2005 
37. Dimitriu P, Brandon I, Kausar M, Hilary L, Mohn WW, Hillebrand GG. New insights into the intrinsic and extrinsic factors that shape the human skin microbiome. mBio. (2021) 10:e00839-19. doi: 10.1128/mBio.00839-19

38. Miranda-Miranda E, Cossio-Bayugar R, Quezada-Delgado MR, SachmanRuiz B, Reynaud-Garza E. "Staphylococcus saprophyticus causa infeccion letal en la garrapata del ganado Rhipicephalus microplus," in: Estrada Venegas EG, Equihua Martinez A, Chaires Grijalva MP, Acuña Soto JA, Padilla Ramirez JR, Mendoza Estrada A, Editors. Entomología Mexicana. México: Sociedad Mexicana de Entomología A.C. (2009) p. 104-8.

39. Miranda-Miranda E, Cossio-Bayugar R, Quezada-Delgado MDR, SachmanRuiz B, Reynaud E. Staphylococcus saprophyticus is a pathogen of the cattle tick Rhipicephalus (Boophilus) microplus. Biocontrol Sci Technol. (2010) 20:1055-67. doi: 10.1080/09583157.2010.505325

40. Klyachko O, Stein BD, Grindle N, Clay K, Fuqua C. Localization and visualization of a coxiella-type symbiont within the lone star tick, Amblyomma americanum. Appl Environ Microbiol. (2007) 73:658494. doi: 10.1128/AEM.00537-07

41. Duron O, Morel O, Noël V, Buysse M, Binetruy F, Lancelot R, et al. Tickbacteria mutualism depends on B vitamin synthesis pathways. Curr Biol. (2018) 28:1896-902.e5. doi: 10.1016/j.cub.2018.04.038

42. Narasimhan S, Rajeevan N, Liu L, Zhao YO, Heisig J, Pan J, et al. Gut microbiota of the tick vector Ixodes scapularis modulate colonization of the Lyme disease spirochete. Cell Host Microbe. (2014) 15:5871. doi: 10.1016/j.chom.2013.12.001

43. Amoo AO, Dipeolu OO, Akinboade AO, Adeyemi A. Bacterial isolation from and transmission by Boophilus decoloratus and Boophilus geigyi. Folia Parasitol (Praha). (1987) 34:69-74.

44. Adegoke A, Kumar D, Bobo C, Rashid MI, Durrani AZ, Sajid MS, et al. Tick-borne pathogens shape the native microbiome within tick vectors. Microorganisms. (2020) 8:1299. doi: 10.3390/microorganisms 8091299

45. Macaluso KR, Sonenshine DE, Ceraul SM, Azad AF. Rickettsial infection in Dermacentor variabilis (Acari: Ixodidae) inhibits transovarial transmission of a second Rickettsia. J Med Entomol. (2002) 39:809-13. doi: 10.1603/0022-2585-39.6.809

46. Gall CA, Reif KE, Scoles GA, Mason KL, Mousel M, Noh SM, et al. The bacterial microbiome of Dermacentor andersoni ticks influences pathogen susceptibility. ISME J. (2016) 10:1846-55. doi: 10.1038/ismej.2015.266

47. Heisig M, Abraham NM, Liu L, Neelakanta G, Mattessich S, Sultana H, et al. Antivirulence properties of an antifreeze protein. Cell Rep. (2014) 9:417-24. doi: 10.1016/j.celrep.2014.09.034

48. Abraham NM, Liu L, Jutras BL, Yadav AK, Narasimhan S, Gopalakrishnan V, et al. Pathogen-mediated manipulation of arthropod microbiota to promote infection. Proc Natl Acad Sci USA. (2017) 114:E781-90. doi: 10.1073/pnas.1613422114

49. Segura JA, Isaza JP, Botero LE, Alzate JF, Gutiérrez LA. Assessment of bacterial diversity of Rhipicephalus microplus ticks from two livestock agroecosystems in Antioquia, Colombia. PLoS ONE. (2020) 15:e0234005. doi: 10.1371/journal.pone.0234005

50. de la Fuente J, Antunes S, Bonnet S, Cabezas-Cruz A, Domingos AG, Estrada-Peña A, et al. Tick-pathogen interactions and vector competence: identification of molecular drivers for tick-borne diseases. Front Cell Infect Microbiol. (2017) 7:114. doi: 10.3389/fcimb.2017.00114

51. Greay TL, Gofton AW, Paparini A, Ryan UM, Oskam CL, Irwin PJ. Recent insights into the tick microbiome gained through next-generation sequencing. Parasit Vectors. (2018) 11:12. doi: 10.1186/s13071-017-2550-5

52. Thoendel M. targeted metagenomics offers insights into potential tick-borne pathogens. J Clin Microbiol. (2020) 58:e01893-20. doi: 10.1128/JCM.01893-20

53. Karim S, Budachetri K, Mukherjee N, Williams J, Kausar A, Hassan MJ, et al. A study of ticks and tick-borne livestock pathogens in Pakistan. PLoS Negl Trop Dis. (2017) 11:1-17. doi: 10.1371/journal.pntd.0005681
54. Rojas-Jaimes J, Lindo-Seminario D, Correa-Núñez G, Diringer B Characterization of the bacterial microbiome of Rhipicephalus (Boophilus) microplus collected from Pecari tajacu "Sajino" Madre de Dios, Peru. Sci Rep. (2021) 11:6661. doi: 10.1038/s41598-021-86177-3

55. Hernández-Jarguín A, Díaz-Sánchez S, Villar M, de la Fuente J. Integrated metatranscriptomics and metaproteomics for the characterization of bacterial microbiota in unfed Ixodes ricinus. Ticks Tick Borne Dis. (2018) 9:124151. doi: 10.1016/j.ttbdis.2018.04.020

56. Jiao J, Lu Z, Yu Y, Ou Y, Fu M, Zhao Y, et al. Identification of tick-borne pathogens by metagenomic next-generation sequencing in Dermacentor nuttalli and Ixodes persulcatus in Inner Mongolia, China. Parasit Vectors. (2021) 14:287. doi: 10.1186/s13071-021-04740-3

57. Obregón D, Bard E, Abrial D, Estrada-Peña A, Cabezas-Cruz A. Sex-specific linkages between taxonomic and functional profiles of tick gut microbiomes. Front Cell Infect Microbiol. (2019) 9:298. doi: 10.3389/fcimb.2019.00298

58. Villar M, Ayllón N, Alberdi P, Moreno A, Moreno M, Tobes R, et al. Integrated metabolomics, transcriptomics and proteomics identifies metabolic pathways affected by anaplasma phagocytophilum infection in tick cells*[S]. Mol Cell Proteomics. (2015) 14:3154-72. doi: 10.1074/mcp.M115.051938

59. Wilke ABB, Marrelli MT. Paratransgenesis: a promising new strategy for mosquito vector control. Parasit Vectors. (2015) 8:342. doi: 10.1186/s13071-015-0959-2

60. Ben Beard C, Cordon-Rosales C, Durvasula RV. Bacterial symbionts of the triatominae and their potential use in control of Chagas disease transmission. Annu Rev Entomol. (2002) 47:123-141. doi: 10.1146/annurev.ento.47.091201.145144

61. Riehle MA, Jacobs-Lorena M. Using bacteria to express and display antiparasite molecules in mosquitoes: current and future strategies. Insect Biochem Mol Biol. (2005) 35:699-707. doi: 10.1016/j.ibmb.2005.02.008

62. de la Fuente J, Almazan C, Canales M, Perez de la Lastra JM, Kocan KM, Willadsen P. A ten-year review of commercial vaccine performance for control of tick infestations on cattle. Anim Heal Res Rev. (2007) 8:238. doi: 10.1017/S1466252307001193

63. Ndawula CJ, Tabor AE. Cocktail anti-tick vaccines: the unforeseen constraints and approaches toward enhanced efficacies. Vaccines. (2020) 8:457. doi: $10.3390 /$ vaccines 8030457

64. Rodríguez-Camarillo SD, Quiroz-Castañeda RE, Aguilar-Díaz H, Vara-Pastrana JE, Pescador-Pérez D, Amaro-Estrada I, et al. Immunoinformatic analysis to identify proteins to be used as potential targets to control bovine anaplasmosis. Int $J$ Microbiol. (2020) 2020:8882031. doi: 10.1155/2020/8882031

Conflict of Interest: The authors declare that the research was conducted in the absence of any commercial or financial relationships that could be construed as a potential conflict of interest.

Publisher's Note: All claims expressed in this article are solely those of the authors and do not necessarily represent those of their affiliated organizations, or those of the publisher, the editors and the reviewers. Any product that may be evaluated in this article, or claim that may be made by its manufacturer, is not guaranteed or endorsed by the publisher.

Copyright (C) 2021 Aguilar-Díaz, Quiroz-Castañeda, Cobaxin-Cárdenas, SalinasEstrella and Amaro-Estrada. This is an open-access article distributed under the terms of the Creative Commons Attribution License (CC BY). The use, distribution or reproduction in other forums is permitted, provided the original author(s) and the copyright owner(s) are credited and that the original publication in this journal is cited, in accordance with accepted academic practice. No use, distribution or reproduction is permitted which does not comply with these terms. 